acima do ombro que podem ser efetuados até 40.000 vezes por ano num jogador profissional. ${ }^{7}$ Consistem nas fases de

\title{
m Biomecânica e Traumatologia ఠ do Voleibol
}

Dr. Filipe Rodrigues ${ }^{1}$, Dr. Pedro Neves ${ }^{2}$, Dr. Diogo Lino Moura ${ }^{3}$

${ }^{1}$ Médico Interno Complementar de Ortopedia do Centro Hospitalar e Universitário do Porto (CHUP); ${ }^{2}$ Assistente Hospitalar de Ortopedia do CHUP; ${ }^{3}$ Assistente Hospitalar de Ortopedia do Centro Hospitalar e Universitário de Coimbra, Faculdade de Medicina da Universidade de Coimbra.

\section{RESUMO / ABSTRACT}

O voleibol é uma das modalidades desportivas mais praticadas no Mundo, reunindo cerca de 500 milhões de praticantes. Apesar de ser considerada uma modalidade sem contacto físico, na qual as equipas se encontram separadas por uma rede, os atletas estão sujeitos a lesões traumáticas e de sobreuso de perfil único relacionadas com as características biomecânicas desta modalidade. Trata-se de um desporto overhead, isto é, com movimentos frequentes dos membros superiores acima do nível da cabeça, o que traz consequências em termos da prevalência de patologias relacionadas com o ombro. A repetição de saltos, rotações do tronco e rápidas mudanças de direção predispõem a lesões da região lombar, joelhos e tornozelos. De modo a elaborar estratégias para a prevenção destas lesões torna-se imprescindível conhecer a incidência, o tipo e os mecanismos das lesões mais frequentes do voleibol.

Volleyball is one of the most popular sports in the world, practiced by 500 million players worldwide. Although considered a non-contact sport in which teams are separated by a net, athletes are prone to traumatic and overuse injuries with unique profile related to the biomechanical characteristics of the sport. It is considered an overhead sport due to the frequent movements of the upper limbs above the level of one's head, which has consequences in terms the prevalence of shoulder conditions. Repeated jumps, trunk rotations and quick direction changes predispose to lower back, knee and ankle injuries. In order to develop strategies for injury prevention it is essential to know the incidence, type and mechanisms of the most frequent volleyball injuries.

\section{PALAVRAS-CHAVE / KEYWORDS}

Voleibol, lesões traumáticas, ombro, cotovelo, punho, sobreuso Volleyball, traumatic injuries, shoulder, elbow, wrist, overuse

\section{Introdução}

O voleibol foi criado originalmente como uma atividade recreativa pelo nova-iorquino William G. Morgan (1870-1942), diretor de Educação Física na YMCA de Massachusetts, que originalmente a batizou de Mintonette. Após a demonstração inaugural da modalidade em 1896, Alfred T. Halstead, Professor do Springfield College, propôs a alteração do nome para volleyball. ${ }^{1}$

A Federação Internacional de Voleibol (FIVB) estima que atualmente a modalidade seja praticada por cerca de 500 milhões de pessoas em todo o Mundo, integrando os Jogos Olímpicos desde 1964. ${ }^{2,3}$

Apesar de ser considerada uma modalidade sem contacto físico, na qual as equipas se encontram separadas por uma rede, os atletas estão sujeitos a lesões traumáticas e de sobreuso de perfil único. ${ }^{4}$ As lesões agudas, nomeadamente entorses dos tornozelos e dedos das mãos, são mais frequentes. As lesões de sobreuso comprometem maioritariamente os ombros e os joelhos. ${ }^{5}$

\section{Biomecânica do voleibol}

No voleibol são realizados múltiplos tipos de arremessos, cada um possuindo diferentes fatores biomecânicos que contribuem para o espetro de lesão. Os mais frequentes são: ${ }^{6}$

- o passe (Figura 1) - tocar na bola apenas com as pontas dos dedos, fazendo um triângulo através da junção dos polegares e indicadores de ambas as mãos,

- a manchete (Figura 2) - membros superiores juntos, com extensão dos cotovelos e punhos, de forma a receber a bola com o máximo de controlo possível,

- o ataque (Figura 3),

- o bloqueio (Figura 4) e

- o serviço (Figura 5), mecanicamente semelhante ao ataque. O serviço e o ataque contemplam movimentos altamente técnicos
- preparação (abdução do braço superior a $90^{\circ}$ e rotação lateral do ombro),

- aceleração (súbita rotação medial e adução do braço até atingir a bola, podendo a velocidade da mão alcançar os $120 \mathrm{~km} / \mathrm{h})^{8} \mathrm{e}$

- a desaceleração (após o impacto com a bola o ombro continua a sua rotação medial e adução do braço, de modo a dissipar a restante energia cinética não transferida para a bola).
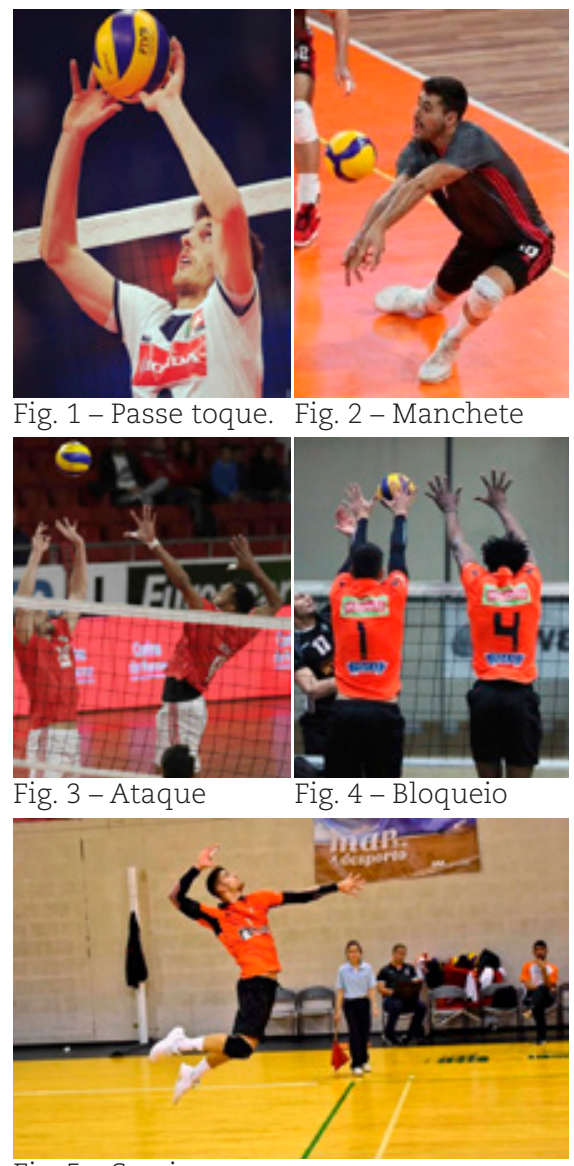

Fig. 5 - Serviço

O voleibol provoca stress repetitivo de grande intensidade sobre o ombro, sujeitando este complexo articular a amplitudes limite diversas vezes durante o treino ou jogo. Os movimentos repetidos de elevação anterior, abdução e rotação lateral do ombro, frequentes durante o serviço e arremessos, provocam de forma progressiva aumento da rotação lateral do ombro e subluxação anterior da cabeça do úmero. O aumento da rotação lateral induz contratura da cápsula articular posterior e da banda posterior do ligamento glenoumeral inferior, o que tem como 
consequência o défice da rotação medial da articulação glenoumeral. O ombro dominante tem maior risco de lesão quando a perda da rotação medial é igual ou superior a $18^{\circ}$ e há uma diferença igual ou superior a $5^{\circ}$ na amplitude total de movimentos entre ambos os ombros. ${ }^{9}$

\section{Traumatologia do voleibol}

Bere T. et al estudaram as lesões registadas pela Federação Internacional de Voleibol em todos os grandes torneios (Campeonato Mundial, Copa do Mundo, World Grand Prix, Jogos Olímpicos, Liga Mundial) decorridos entre setembro de 2010 e novembro de 2014, num total de 25633 lesões. A incidência de lesões durante os jogos que levaram a incapacidade de prosseguir com o jogo foi de 3.8/1000 horas de jogo, não se tendo verificado diferença estatisticamente significativa entre géneros. As áreas do corpo mais frequentemente lesionadas foram os tornozelos (25.9\%), seguidos dos joelhos (15.2\%), dedos das mãos (10.7\%), coluna lombar (8.9\%) e ombro (5\%). Os tipos de lesões mais frequentemente diagnosticadas foram as entorses (32.5\%), nomeadamente do tornozelo, dedos das mãos e joelhos, as roturas musculares parciais (14.1\%), sobretudo ao nível da região lombar e da coxa, e as contusões (12.7\%). ${ }^{4}$ Por sua vez, Pollar KA. et al registaram as lesões associadas ao voleibol em idade pediátrica apresentadas aos serviços de urgência dos Estados Unidos da América (com base num serviço eletrónico nacional comum a 100 hospitais), entre 1990 e 2009. Registou-se uma taxa de internamento de 1\%. Cerca de $48 \%$ das lesões ocorreram nos membros superiores (cerca de metade nos dedos das mãos), 39\% nos membros inferiores (maioritariamente ao nível dos tornozelos), 9\% na cabeça ou pescoço e $4 \%$ no tronco. Os tipos de lesões mais frequentes foram as roturas musculares ou ligamentares parciais (54\%) e as fraturas e luxações (17\%). ${ }^{10}$

Alguns trabalhos identificaram o género como fator de risco para algumas lesões, a realçar um aumento na prevalência de tendinopatia patelar nos homens e de subluxação da articulação glenoumeral nas mulheres. ${ }^{11,12}$

A função da coifa dos rotadores é de limitar movimentos excessivos e potencialmente lesivos da cabeça do úmero na cavidade glenoideia, bem como de auxiliar nas mobilidades do ombro. O desequilíbrio entre os rotadores mediais e os rotadores laterais da coifa dos rotadores, associado a laxidão assimétrica dos ligamentos capsulares e do labrum glenoideu, favorecem a instalação de instabilidade glenoumeral, possibilitando movimentos translacionais excessivos e possivelmente lesivos da cabeça do úmero sobre a cavidade glenoideia. ${ }^{2} \mathrm{O}$ conflito interior do ombro surge como resultado da subluxação anterior da cabeça do úmero nos movimentos extremos de elevação anterior, abdução e rotação lateral do ombro frequentemente executados no voleibol. Este conflito verifica-se entre a face articular dos tendões supra e infraespinhoso da coifa e a porção póstero-superior do rebordo glenoideu e labrum, podendo originar tendinopatia da coifa dos rotadores, espetro que pode variar entre tendinites, tendinoses e rotura tendinosa. As forças de cisalhamento aplicadas sobre a porção superior do labrum glenoideu, proporcionadas pelos característicos movimentos overhead do voleibol, favorecem a sua rotura. Neste desporto são típicas as roturas tipo SLAP (superior labral tear from anterior to posterior), que são localizadas na porção superior do labrum ao nível do local de inserção do tendão da longa porção do bicípite braquial no próprio labrum. De acordo com o seu padrão, estas roturas podem ou não também afetar o tendão da longa porção do bicípite braquial. ${ }^{13,14}$

A prevalência da neuropatia do nervo supraescapular é de 12.5 - 33\% em jogadores de voleibol de alta competição. ${ }^{15}$ É caracterizada por diminuição de força de abdução e rotação lateral do braço devido a desnervação dos músculos supra e infraespinhoso. O nervo supraescapular pode ser comprimido a nível do canal supraescapular, originando atrofia do supra e infraespinhoso, mas nos jogadores de voleibol é mais frequente a sua compressão no canal espinoglenoideu, causando atrofia isolada do infraespinhoso. ${ }^{16}$ A sua patogénese ainda não se encontra totalmente esclarecida. Múltiplos microtraumas causados por tração repetida ou microêmbolos dos vasa nervorum do nervo supraescapular são possíveis causas. O nervo supraescapular também pode ser comprimido no canal espinoglenoideu por um quisto sinovial consequente a uma rotura do labrum posterior. ${ }^{15}$ A eletromiografia é o exame complementar que confirma as suspeitas clínicas de diagnóstico desta síndrome.. ${ }^{17}$ No diagnóstico diferencial da neuropatia do nervo supraescapular deve fazer parte a síndrome de Parsonage-Turner (neurite do plexo braquial). ${ }^{2}$

A síndrome do espaço quadrilátero é uma entidade rara causada pela compressão do nervo axilar e artéria umeral circunflexa posterior ao nível do espaço quadrilátero. Esta compressão é habitualmente agravada aquando dos movimentos de abdução e rotação lateral dos braços, que ao condicionarem a subida dos limites inferiores do espaço quadrilátero (longa porção do tricípete braquial e teres major), provocam redução deste espaço e compressão do seu conteúdo. ${ }^{18}$ Este síndrome é muitas vezes atribuído a bandas fibrosas e aderências no espaço quadrilátero que se formam por prováveis microtraumas locais repetidos. As manifestações clínicas do síndrome incluem omalgia difusa, atrofia, parestesias e parésia ao nível do deltóide e do teres minor, com diminuição da rotação lateral do ombro. Pode também apresentar manifestações vasculares, tais como isquémia digital devido a embolização arterial, sendo que todos os sintomas agravam na abdução e rotação lateral dos braços. ${ }^{19-21}$

\section{As lesões dos dedos das mãos são} comuns no voleibol. São frequentes durante os bloqueios, por carga axial exercida sobre a extremidade dos dedos e hiperextensão dos mesmos após contacto com a bola, podendo também ocorrer na fase de desaceleração de um ataque, onde são frequentes impactos dos dedos com a rede ou outro jogador. A maioria das lesões são ligamentares e ocorrem ao nível da articulação interfalângica proximal. Estão também descritas lesões dos tendões extensores dos dedos, nomeadamente situações de dedo em martelo decorrente de trauma axial sobre a ponta dos dedos. ${ }^{2}$

O movimento repetido de manchetes, em particular se a bola for recebida de modo incorreto na porção volar radial dos antebraços, pode conduzir a situações de sinovite da bainha dos tendões do primeiro compartimento 
extensor dos dedos (curto extensor do polegar e longo abdutor do polegar), condição denominada tenossinovite de De Quervain.22

As lesões da coluna lombar podem contemplar até $14 \%$ do total das lesões do voleibol. ${ }^{23}$ Estas devem-se a repetidos e muitas vezes assimétricos movimentos de rotação, flexão e inclinação lateral do tronco, típicos dos atacantes no voleibol. ${ }^{24} \mathrm{~A}$ repetida flexão assimétrica do tronco está associada a roturas parciais dos músculos paravertebrais, a roturas ligamentares da coluna e a discopatias, nomeadamente hérnias discais e discopatia degenerativa, raramente condicionando neste desporto compressão sintomática de nervos espinhais..$^{23,25} \mathrm{O}$ movimento repetido de hiperextensão da coluna lombar durante o serviço ou ataque condiciona elevadas forças de tensão sobre a pars interarticularis, em particular da vértebra de L5, podendo condicionar situações de espondilólise, com eventual progressão para espondilolistese. Os adolescentes são mais vulneráveis a este tipo de lesões, pois têm pars interarticularis mais frágeis. ${ }^{2}$

A tendinopatia patelar está habitualmente associada aos repetidos saltos realizados de forma explosiva. Estima-se que $40 \%$ a $50 \%$ dos jogadores de alta competição venha a desenvolver pelo menos um episódio de tendinopatia patelar. ${ }^{23,26} \mathrm{~A}$ dor localiza-se mais frequentemente na porção proximal do tendão patelar, junto à sua inserção na patela. Também podem ocorrer situações de tendinose e, eventualmente, de rotura do tendão patelar. ${ }^{2}$

As entorses do tornozelo são as lesões agudas mais frequentes do voleibol. Ocorrem maioritariamente na zona de conflito, perto da rede, quando após um bloqueio o pé do defesa aterra sobre o pé do atacante. ${ }^{2}$ As recidivas de entorses do tornozelo são comuns neste desporto, atingindo por vezes a incidência de $42 \%$ nos 6 meses após a entorse inicial. ${ }^{27}$

As cargas excêntricas impostas pelos saltos e aterragens neste desporto podem também originar inflamação do paratendão do tendão de Aquiles. As repetições destas mesmas cargas podem conduzir a tendinopatia crónica de difícil tratamento e, eventualmente, a rotura do tendão de Aquiles degenerado. ${ }^{2}$

\section{Conclusão}

O voleibol é uma das modalidades desportivas mais praticadas no Mundo. Apesar de ser considerada uma modalidade sem contacto físico na qual os jogadores se encontram separados por uma rede, devido às características biomecânicas desta modalidade os atletas estão sujeitos a lesões traumáticas e de sobreuso de perfil especial. É essencial conhecer a incidência e os mecanismos das lesões mais frequentes do voleibol, assim como fatores individuais, de forma a criar estratégicas para a sua prevenção durante a prática desportiva.

Um agradecimento especial ao atleta Afonso Guerreiro por ter facultado as fotografias.

Os autores declaram não haver conflitos de interesse ou económicos, assim como a originalidade do manuscrito e a sua não publicação prévia

\section{Correspondência:}

Dr. Filipe Rodrigues

Serviço de Ortopedia do Centro Hospitalar e Universitário do Porto, Porto filipejrod@hotmail.com

\section{Bibliografia}

1. https://www.fivb.org/TheGame/Volleyball_Story.htm.

2. Eerkes K. Volleyball Injuries. Curr Sports Med Rep. 2012; 11(5):251-6.

3. Wang HK. A Descriptive Epidemiological Study of Shoulder Injury in Top Level English Male Volleyball Players. Int J Sports Med. 2001 Dec 31; 22(02):159-63.

4. Bere T, Kruczynski J, Veintimilla N, Hamu Y, Bahr R. Injury risk is low among world-class volleyball players: 4-year data from the FIVB Injury Surveillance System. Br J Sports Med. 2015 Sep; 49(17):1132-7.

5. Cuñado-González Á, Martín-Pintado-Zugasti A, Rodríguez-Fernández ÁL. Prevalence and Factors Associated With Injuries in Elite Spanish Volleyball. J Sport Rehabil. 2019 Feb 14; 1-7.

6. Santini J. Voleibol escolar: da iniciação ao treinamento. Editora da ULBRA 2008 Jan. capitulo IV, 47.

7. Dubotzky V, Leistner M. Volleyball. In: Ballreich $R$, Kuhlow-Balireich $A$, eds. Biomechanik der Sportarten: Bd. 3: Biomechanik der Sportspiele, vol 2. Stuttgart: Enke Verlag 1992; 72-123.

8. Stickley CD, Hetzler RK, Freemyer BG, Kimura IF. Isokinetic peak torque ratios and shoulder injury history in adolescent female volleyball athletes. J Athl Train. 2008; 43(6):571-577.

9. Challoumas D, Stavrou A, Dimitrakakis G. The volleyball athlete's shoulder: biomechanical adaptations and injury associations. Sports Biomech. 2017 Apr 3; 16(2):220-37.
10. Pollard KA, Shields BJ, Smith GA. Pediatric Volleyball-Related Injuries Treated in US Emergency Departments, 1990-2009. Clin Pediatr (Phila). 2011 Sep; 50(9):844-52.

11. Visnes, H.; Bahr, R. Training volume and body composition as risk factors for developing jumper's knee among young elite volleyball players. Scand. J. Med. Sci. Sports 2012; 23:607-613.

12. Mjaanes, J.M.; Briner, W.W. Trends in shoulder injuries among elite volleyball players in the USA. Br. J. Sports Med. 2005; 39, 373-408.

13. Reeser JC, Joy EA, Porucznik CA, Berg RL, Colliver EB, Willick SE. Risk Factors for Volleyball-Related Shoulder Pain and Dysfunction. PM\&R. 2010 Jan;2(1):27-36.

14. Seminati E, Minetti AE. Overuse in volleyball training/practice: A review on shoulder and spine-related injuries. Eur J Sport Sci. 2013 Nov; 13(6):732-43.

15. Pieber K, Herceg M, Fialka C, Oberleitner G, Gruther W, Paternostro-Sluga T. Is suprascapular neuropathy common in high-performance beach volleyball players? A retrospective analysis. Wien Klin Wochenschr. 2014 Oct; 126(19-20):655-8.

16. Thompson WA, Kopell HP. Peripheral entrapment neuropathies of the upper extremity. $\mathrm{N}$ Engl J Med. 1959; 260:1261-5.

17. Boykin RE, Friedman DJ, Higgins LD, et al. Suprascapular neuropathy. J Bone Joint Surg Am. 2010; 92:2348-64.

18. Cahill BR, Palmer RE. Quadrilateral space syndrome. J Hand Surg 1983; 8:65-69.

19. Brown SA. et al. Quadrilateral Space Syndrome: The Mayo Clinic Experience With a New Classification System and Case Series. Mayo Clin Proc. 2015; 90(3):382-394.

20. Atema JJ, Ünlü Ç, Reekers JA, Idu MM. Posterior Circumflex Humeral Artery Injury with Distal Embolisation in Professional Volleyball Players: A Discussion of Three Cases. Eur J Vasc Endovasc Surg. 2012 Aug; 44(2):195-8.

21. Reekers JA, den Hartog BMG, Kuyper CF, Kromhout JG, Peeters FLM. Traumatic Aneurysm of the Posterior Circumflex Humeral Artery: A Volleyball Player's Disease? J Vasc Interv Radiol. 1993 May; 4(3):405-8.

22. Rossi C, Cellocco P, Margaritondo E, Bizzarri F, Costanzo G. De Quervain Disease in Volleyball Players. Am J Sports Med. 2005 Mar; 33(3):424-7.

23. Briner WW, Kacmar L. Common Injuries in Volleyball: Mechanisms of Injury, Prevention and Rehabilitation. Sports Med. 1997 Jul; 24(1):65-71.

24. Dalichau, S., \& Scheele, K. Die thorakolumbale wirbelsa ülenform männlicher leistungsvolleyballspieler [The thoracolumbar spinal curvature in male competitive volleyball players]. Deutsche Zeitschrift fur Sportmedizin, 2002; 53(1):12-16.

25. Bartolozzi C. et al. The incidence of disk changes in volleyball players. The magnetic resonance findings. Radiol Med. 1991; 82:757-760.

26. Lian O, Engebretsen L, Ovrebo RV, Bahr R. Characteristics of leg extensors in male volleyball players with jumper's knee. Am. J. Sports Med. 1996; 24:380Y5.

27. Bahr R, Bahr I. Incidence of acute volleyball injuries: a prospective cohort study of injury mechanisms and risk factors. Scand. J. Med. Sci. Sports. 1997; 7:166Y71. 